\title{
Los medios en la gestión de la comunicación de las ONG en tiempos de crisis: oportunidades para el cambio social
}

\section{The media in communication management of NGO in times of crisis: opportunities for social change}

\author{
Elisa Regadera González*, Hildegart González Luis**, Pilar Paricio Esteban*** \\ * International University of Catalonia, España \\ ** University of Navarra, España \\ *** Universidad CEU Cardenal Herrera, España
}

Resumen

El artículo estudia cómo ha influido la crisis económica en la gestión de la comunicación entre las ONG y los medios. También analiza las oportunidades informativas generadas, que estas entidades podrían utilizar para contribuir al cambio social: visibilizar el aumento producido en las necesidades a cubrir; evidenciar la reducción de ingresos a la que se han visto sometidas, y denunciar las desigualdades sociales para conseguir el apoyo de la opinión pública.

El estudio se centra en la Comunidad Valenciana -una de las más castigadas por la crisis- y desarrolla una triple metodología: entrevistas a los responsables de comunicación de una muestra representativa de ONG que atienden a sectores de población vulnerables en Valencia; análisis del tratamiento periodístico que han recibido en 2008 (inicio de la crisis) y 2012 (consolidación de la crisis) en los cinco diarios de referencia de Valencia; y entrevistas a los cinco periodistas responsables de la cobertura de temas sociales y ONG en los diarios analizados.

Los resultados obtenidos permitirán comprobar la influencia de la crisis en la gestión de la comunicación de las ONG y en el tratamiento periodístico de estas entidades. Además han facilitado la identificación de buenas prácticas entre ONG y medios de comunicación, encontrando un mayor compromiso en los medios hacia la denuncia de las injusticias potenciadas por la crisis, mientras que las ONG se han centrado más en la divulgación de su labor.

Palabras clave: ONG, Departamentos de Comunicación, cambio social, gabinete de prensa, gestión informativa, encuadre.

Abstract

This article explains how the economical crisis has influenced in the communication management between NGO's and the media. It also analyses the informative opportunities that the aforementioned entities could use for contributing to the social transformation and therefore, give greater visibility to the increasing needs to be fulfilled; making evident the reduction of the incomes that they have suffered, and reporting the social inequalities trying get the support of the public opinion.

This analysis is focused in the "Comunidad Valenciana"- one of the regions hardest hit by the crisis- and develops a triple methodology: interviews to communication responsibles from some NGOs who help vulnerable segments of population in Valencia; content analysis of journalist treatment received in 2008 (from the beginning of the crisis) and 2012 (crisis consolidation) in five different relevant newspapers in Valencia and interviews to the five journalists responsible of the social matters and NGOs in those newspapers studied.

The results obtained will let test the influence of the crisis in the NGOs communication management and in the journalist treatment of the third sector organizations. It has also facilitated the identification of best practices in NGOs and the media finding a greater commitment in the media denouncing the injustices enhanced by the crisis, while NGOs have focused on the dissemination of its work.

Keywords: NGO, Communicative Departments, social change, press office, informative management, frame. 


\section{Introducción}

Exclusión social y pobreza constituyen problemas crecientes en los países europeos. Desde el 2007, España ha estado inmersa en una crisis económica que ha puesto en aprietos a porcentajes cada vez mayores de la población. En 2012, el 21,1\% de los españoles se encontraba en situación de pobreza (Sánchez Morales, 2013: 51) y según el Instituto Nacional de Estadística (INE 2013: 28), España tenía ese año un 25\% de desempleo, la cifra más elevada de la Unión Europea. Son varios los autores que apuntan que el paro de larga duración, junto al endeudamiento en la adquisición de viviendas y la reducción del gasto social (40\% menos en 2013 para servicios sociales básicos), han sido los principales desencadenantes del tránsito que muchas personas y familias han realizado hacia la pobreza y la exclusión social (Sánchez, 2013; Gutiérrez, 2013; Belzunegui, 2012).

Esta situación ha dado lugar a la aparición de los "nuevos pobres", personas sin antecedentes de exclusión ni desadaptación que, como consecuencia de la crisis, se han visto abocados -según el Observatorio de la Realidad Social de Cáritas Española- primero a buscar apoyo en sus familias, seguido del trabajo en la economía sumergida, y en último lugar, a la búsqueda de apoyo por parte de organizaciones sociales (Caritas 2011: 12).

La recesión económica ha enfrentado a las ONG a dos movimientos de signo contrario: a un incremento de la demanda de sus servicios y a una disminución de sus recursos financieros (OTS, 2009). La disminución de recursos económicos se debe a las fuertes restricciones presupuestarias de las Administraciones Públicas (Fundación Luis Vives, 2013); a la desaparición de las cajas de ahorros -financiadores tradicionales del sector- y a la caída de altas de nuevos socios (ESADE, PWC \& Fundación La Caixa, 2013). A partir del 2012, esta situación ha conllevado reducciones de plantillas; desaparición de entidades o sedes; mayor competencia por los fondos e inicio de procesos de fusión (CONGDE, 2014).

En este contexto, diversos autores se cuestionaban el futuro del sector y apuntaban los retos a los que se enfrentará (Gómez, 2013; Martínez, 2013; Ruiz-Giménez 2013). También se han publicado artículos que reflexionaban sobre los nuevos modelos de comunicación que debieran desarrollar las ONG en este nuevo marco mundial en el que los movimientos sociales han adquirido mayor protagonismo y las nuevas tecnologías abren oportunidades (Pinazo \& Nos, 2013; Marí, 2013; Marfil 2013; Arroyo \& Baños, 2013a; Arroyo \& Baños 2013b; Arroyo; Baños \& Van-Wyck 2013c; Nos, Iranzo \& Farné, 2012; Ramil, 2012; Soria, 2011; Duran \& Fernández, 2010; Baraybar, 2009). Sin embargo, otras publicaciones advertían sobre el riesgo que corría la comunicación en estas entidades, ya que las acciones destinadas a sensibilizar a la sociedad civil podían experimentar un retroceso en favor de proyectos de atención (OTS, 2009; ESADE, PWC \& Fundación La Caixa, 2013).

Entre los múltiples cambios experimentados por la comunicación de las ONG en los últimos años, este artículo se centra en la relación de estas entidades con los medios de comunicación tradicionales durante la crisis económica. Desde un planteamiento teórico, parece lógico sugerir que su gestión comunicativa podría adquirir una relevancia mayor, pudiendo ser empleada por las ONG para visibilizar el aumento de las necesidades que cubren y la reducción de sus ingresos, o para denunciar las desigualdades sociales y movilizar a sectores de población en su compromiso con el cambio social. Además, podría ser muy probable que, en este contexto, los medios acudieran más a ellas como fuentes, puesto que sus acciones adquieren mayor interés noticioso. 
A la hora de abordar el estado de la cuestión, partiendo de investigaciones previas, delimitaremos la situación de los departamentos de comunicación en las ONG y de su relación con los medios.

\section{Hipótesis y objetivos}

Las hipótesis de partida de la investigación son las siguientes:

1. Presuponemos que la crisis económica habrá influido en la gestión de la comunicación desarrollada por las Organizaciones del Tercer Sector y en sus relaciones con los medios, siendo considerados un stakeholder clave en su estrategia comunicativa, ya que en un entorno adverso donde se reducen los recursos y aumentan las necesidades, los medios les permitirín llegar a públicos amplios con un coste menor que el requerido por técnicas publicitarias aportando credibilidad a sus mensajes.

2. Presuponemos que la crisis habrá potenciado el tratamiento periodístico de las ONG provocando cambios en el modelo informativo: mayor cobertura de estas entidades y generación de oportunidades que podrían ser aprovechadas por las ONG para proyectar su labor con los colectivos más vulnerables y para denunciar las desigualdades potenciadas por las crisis.

Teniendo en cuenta las hipótesis señaladas, los objetivos generales y específicos son:

1) Conocer el modelo de gestión de la comunicación de las ONG seleccionadas, con particular atención a la importancia concedida a las relaciones con los medios durante la crisis. Para ello, los objetivos específicos son:

1.1. Analizar la modalidad de gestión de la comunicación de los Departamentos de las ONG y su consideración estratégica.

1.2. Comprobar si disponen de un Plan de comunicación y cómo ha repercutido la crisis en sus objetivos.

1.3. Constatar el peso del área de relaciones con los medios en los Departamentos de comunicación y la influencia de la crisis en la gestión de dicha área, así como en los temas y frames priorizados por los Gabinetes.

2) Conocer la relación de los periodistas con las organizaciones del Tercer Sector y la atención prestada a dichas entidades durante la crisis. Para ello, los objetivos específicos son:

2.1. Analizar el interés periodístico por las ONG y valorar si la crisis ha influido en su cobertura.

2.2. Conocer la percepción de los periodistas sobre las ONG como fuentes y si ha variado durante la crisis.

2.3. Conocer los temas solicitados por los medios a los Gabinetes de las ONG, los frame destacados y si han cambiado por la crisis priorizando los mensajes de denuncia y cambio social. 
3) Conocer el tratamiento informativo de las ONG durante la crisis en la prensa de información general en Valencia (años 2008 y 2012). Los objetivos específicos son:

3.1. Conocer la cobertura de las ONG en los medios seleccionados.

3.2. Analizar las fuentes, temas, personajes, presencia de la crisis y encuadre predominante en los medios.

\section{Metodología y muestra}

La presente investigación se orienta a conocer si las organizaciones del Tercer Sector han potenciado su comunicación en los momentos de crisis económica, en un contexto donde los movimientos sociales adquieren un mayor protagonismo. El estudio abordará la gestión de las relaciones con los medios realizada por dichas entidades orientada a visibilizar su papel y movilizar a la sociedad para luchar contra las desigualdades.

En relación a los objetivos de la investigación, se ha utilizado una doble metodología -cualitativa y cuantitativa-, realizando entrevistas y análisis de contenido:

a) En relación al primer y segundo objetivos, se han realizado entrevistas en profundidad (Vallés, 2002) a los responsables de los Gabinetes de comunicación de las ONG y entrevistas semiestructuradas (Piñuel y Gaitán, 1998) a profesionales de los medios, diseñando para ello dos cuestionarios.

b) En cuanto al tercer objetivo, se ha realizado un análisis de contenido cuantitativo de tipo categorial desde la perspectiva del frame (Bardín, 1986; Berganza y Ruiz, 2005; Paricio, Rodríguez y Rabadán, 2012), diseñando un protocolo de análisis con una definición de categorías ad hoc, abordando el análisis de la cobertura, fuente, temas, personajes, frame y presencia de la crisis en los textos.

El estudio analiza la gestión comunicativa de una muestra de organizaciones que han atendido a los sectores de población más vulnerables en los años 2008 (inicio de la crisis) y 2012 (consolidación de la crisis). Además, evaluará el tratamiento periodístico de dichas entidades, lo que permitirá comprobar si los medios les han otorgado un papel más relevante, y encontrar buenas prácticas comunicativas en ONG y medios que faciliten la difusión de la labor desarrollada por estas entidades y la denuncia de las injusticias para contribuir al cambio social.

El estudio se centra en la Comunidad Valenciana, pudiendo ser replicado en otras comunidades. El motivo de su elección es la intensidad con la que ha sufrido la crisis, como confirman diferentes indicadores sobre pobreza y exclusión social. Así, según el VII Informe FOESSA sobre exclusión y desarrollo social en España (2014), los hogares de Valencia se encuentran en una situación de mayor vulnerabilidad (el 64,9\% no están plenamente integrados, frente al 63,1\% en España).

Con el objeto de conseguir una muestra abarcable en relación a los objetivos, la investigación se ha centrado en Valencia, por ser la única provincia de la C.V. en la que algunas ONG cuentan con Departamento de comunicación. Se seleccionaron tres muestras: Departamentos de comunicación de organizaciones del 
Tercer Sector; periodistas de prensa nacional y autonómica y artículos con mención de las ONG en los diarios seleccionados.

Se han elegido seis ONG según los siguientes criterios: que trabajen de manera representativa en Valencia y dispongan de Gabinete de comunicación; que dirijan su trabajo hacia colectivos en riesgo de exclusión, con un elevado número de beneficiarios y con diferentes configuraciones jurídicas. Para identificar las ONG, se realizó una consulta exploratoria a 6 Plataformas de la Comunidad Valenciana (que aglutinan a más de 20 Federaciones y más de 400 entidades), que coincidieron en designar a las siguientes entidades: Cruz Roja, Cáritas, Casa Caridad, Comisión de Ayuda al Refugiado del País Valenciano (CEARPV), Confederación Española de Personas con Discapacidad Física y Orgánica de la Comunidad Valenciana (COCMFECV) y la Federación de Asociaciones en favor de las personas con discapacidad intelectual de la Comunidad Valenciana (FEAPSCV, hoy denominada "Plena Inclusión"). En dichas entidades se ha seleccionado a los responsables de sus Departamento de comunicación.

La muestra de diarios atendió a los siguientes criterios: prensa nacional y local con mayor tirada y difusión en la provincia de Valencia (según OJD en 2008 y 2012) y diverso espectro ideológico. Las cabeceras seleccionadas son: El País, El Mundo, ABC, Levante y Las Provincias. A continuación, se identificó a los periodistas responsables de la cobertura de temas sociales y ONG en 2012 en los cinco diarios.

En cuanto a la muestra temporal, se han elegido los años 2008 y 2012 por considerarlos claves en relación al objeto de estudio. El año 2008 marcó el inicio de la crisis económica en España, ya que, como apuntaban Ortega y Peñalosa (2012), España entró en recesión económica en el segundo trimestre de 2008. Por otra parte, 2012 fue uno de los años centrales de la crisis donde sus consecuencias tuvieron un importante impacto en términos de paro, conflictividad social (Público, 2012) y necesidades de colectivos amplios en situación de vulnerabilidad.

Por consiguiente, como apuntaron Rocha y Aragón (2012), la persistencia en el aumento del desempleo y del desempleo de larga duración - supuso un importante agravamiento de las condiciones de vida y bienestar de la población de 2008 a 2012. Esto se manifestó en diversos indicadores, entre los que cabe destacar la tasa de riesgo de pobreza o exclusión social (indicador estrategia Europa 2020), que desde el inicio de la crisis aumentó en cuatro puntos porcentuales hasta afectar, en 2012, a más 12 millones de personas -en torno al $27 \%$ de la población- (INE, 2012). Se trata por tanto de la época de mayor intensidad en los recortes y donde se produce la mayor erosión de la clase media española. Desde 2013 al momento actual se entra en una etapa de recuperación, más lenta que la producida anteriormente, llegando a valores positivos de la variación interanual del PIB desde principios de 2014 (Plataforma de ONG, 2015).

Esos años marcaron retos importantes en relación a la situación de las ONG, que por un lado y como consecuencia de la intensificación de las crisis, se enfrentaron a un incremento de la demanda de sus servicios por el aumento de las necesidades sociales; y por otro, a una disminución de sus recursos financieros debido a las fuertes restricciones presupuestarias de las Administraciones Públicas, que repercuten directamente en porcentajes elevados.

Ante esta situación, las ONG -desde el inicio de la crisis a su intensificación en 2012- se enfrentaron a cambios estructurales para seguir aportando valor a la sociedad en un momento de especial dificultad, donde la comunicación, y en particular la información en los medios, podría ser un factor clave para visibilizar su papel en la nueva situación, mostrar su impacto social y económico, y sensibilizar a la sociedad española 
sobre la importancia de aportar recursos y apoyos que les ayuden a sacar adelante su misión y los proyectos con los que trabajan.

Analizar la gestión de las relaciones con los medios y la cobertura generada en este periodo puede ser de interés para comprender la evolución en la gestión de la comunicación en estas entidades en los últimos años, a la luz de investigaciones posteriores que subrayaban como han podido contribuir a que las entidades sean cada vez más conscientes de la importancia que tiene la comunicación en su estrategia, lo que queda reflejado en la reducción del año 2015, donde hasta un exiguo 3,2\% de entidades que nunca realizan campañas y acciones de comunicación. Además, algo más de dos tercios de las entidades (69,3\%), percibían que la sociedad tiene una buena imagen de ellas y que confían en el Tercer Sector (Plataforma de ONG, 2015).

En este sentido, se plantea el análisis del tratamiento periodístico de las ONG objeto de estudio en los diarios seleccionados en 2008 y 2012. Para ello, se ha realizado un muestreo aleatorio sistemático aplicando el método de la semana construida (Stempel \& Westley, 1981 y Kayser, 1982) sobre el universo de 2.865 artículos generado del vaciado realizado a través de My news, obteniendo un corpus de 360 unidades informativas. Para tener una referencia de la fiabilidad de los resultados se ha tomado un nivel de significación del 0,05 (alfa) con una precisión del 15\%.

\section{Marco teórico}

La gestión de la comunicación de las ONG. Evolución de los Gabinetes

Si nos aproximamos al estudio de los Departamentos de comunicación de las ONG, encontramos varias tesis doctorales: Fernández, 2004; González, 2005; Balas, 2010; Herranz de la Casa, 2010; González, 2010 y Regadera, 2014. Grosso modo se puede afirmar que todos los investigadores citados, partiendo de diversas muestras de ONGD, abordan el análisis del origen y la evolución de los departamentos de comunicación en estas organizaciones no lucrativas, los rasgos que los definen, los objetivos / finalidades para los que se crearon, los perfiles de los profesionales que trabajan en ellos y las modalidades elegidas por estas instituciones para gestionar su comunicación: departamentos internos, departamentos externos / agencias, o la combinación de ambas modalidades.

Además, existen diversos informes que tratan la situación comunicativa de estas entidades, a nivel nacional o regional (CONGDE, 2007; Fundación Hazloposible Bidea, 2011; Fundar 2011).

Algunas de las conclusiones en las que coinciden las tesis e informes mencionados, que sirven para definir en marco teórico en el que se encuadra este artículo son:

Los primeros Gabinetes de comunicación nacen en España fundamentalmente a partir de mediados de los años 70 del siglo pasado, con un contexto imprescindible para el desarrollo de la comunicación organizacional: la democracia y la economía de mercado. Sin embargo, hasta finales de los años 80 no empiezan a consolidarse en las ONG las áreas dedicadas en exclusiva a la gestión de la comunicación. Fue ya en la década de los 90 cuando las ONG más desarrolladas comienzan a darse cuenta de que sólo siendo transparentes y comunicando de forma clara y estratégica, conseguirían los apoyos necesarios para crear 
una sólida base social y consistencia económica que les permitiera desarrollar su misión de manera sostenible en el tiempo.

Quizá por ello, la mayor parte de los autores que han investigado este ámbito sitúan la consolidación de los Gabinetes de comunicación de las ONG españolas en los 90 (López, 2001; García, 2006; González, 2006). En 1997, el 90\% de las ONG registradas en las CONGDE contaban con un Departamento de comunicación. Los principales motivos que impulsaron la creación de estos Departamentos de comunicación en estas entidades fueron la necesidad de incrementar su conocimiento por parte del público, la toma de conciencia de la importancia que tiene la comunicación en el desarrollo de la misión de las ONG, y la necesidad de agrupar bajo una misma estructura diversidad de funciones.

Con los datos expuestos podemos señalar que se trata de Departamentos con trayectoria corta, lo que podría influir en su nivel de profesionalización. En esa década, las ONG más desarrolladas descubren que sólo comunicando estratégicamente y siendo transparentes, conseguirán los apoyos para crear una base social sólida y ser sostenibles, por lo cual la inclusión de estrategias que dirijan y orienten la comunicación de las ONG, "es también un hecho reciente y en continua mejora. Con frecuencia, dos o tres años después de la creación y consolidación del departamento de comunicación, cada institución incorpora la primera estrategia comunicativa" (González, 2006:79).

A principios del año 2000, según estudios realizados por González (2005:2-3 y 2006:78), los rasgos principales de los Departamentos de comunicación de las ONG españolas eran los siguientes: Juventud; Incremento de la profesionalización y las estrategias; Aumento de recursos adjudicados; Continuo proceso de transformación y mejora, y escasez de recursos humanos en gran parte de las entidades del Tercer Sector.

Todos estos rasgos pueden justificar porqué en la mayoría de los casos, las responsabilidades del departamento de comunicación tienden a concentrarse en una sola persona. El estudio del perfil, de las funciones de los profesionales que desarrollan y ejecutan las acciones desde los Gabinetes de comunicación de las ONG también ha sido objeto de algunas investigaciones (Molina, 2007; Álvarez, 2012).

Sobre el papel determinante de los Dircom en los Gabinetes de comunicación en las ONG, la responsable de Comunicación de la Plataforma del Voluntariado en España Esther Díez, señalaba las siguientes funciones, en una entrevista realizada en el 2006 y recogida por Molina (2007:291): "saber canalizar la marca, implantar políticas de comunicación, elaborar con eficacia e imaginación la planificación de medios, y desarrollar una comunicación interna fluida entre los miembros de la organización, es imprescindible para que la ONG pueda realizar una gestión positiva de su imagen ante la sociedad". Díez aseguraba además que cuando un Dircom hace su trabajo de manera profesional y con medios, la reputación corporativa y la imagen de la ONG sube enteros, lo que permite aumentar los cauces de financiación y conseguir mayor número de objetivos.

Entre los aspectos humanos requeridos por el perfil del Dircom para estas entidades, los profesionales de las ONG coinciden en señalar "la capacidad de imaginación para suplir con ingenio la falta de recursos; la capacidad de negociación para sacar las mejores condiciones a los escasos planes de publicidad; la capacitación docente para seguir enseñando la importancia de la comunicación en la sociedad de la información sin caer en el desaliento; y la paciencia para seguir escuchando aquello de que 'bueno, cuando nos concedan el proyecto pondremos a un/a estudiante de periodismo para que haga las notas de prensa', siempre confundiendo Gabinete de comunicación con Gabinete de prensa" (Molina, 2007:290). 
En relación a las actividades desarrolladas por estos Gabinetes, algunos expertos opinan que sus responsables se dedican mayoritariamente a la gestión de las relaciones con los medios, lo que les aproximaría al modelo de un Gabinete de prensa (Balas, 2011). Pero también es frecuente encontrar numerosas entidades que han evolucionado hacia un Gabinete de comunicación integral que gestiona las relaciones con todos los públicos de interés, y en los que dichas funciones se cubren por varias personas, 0 desde otras áreas (Almansa, 2011). De tal manera que no son pocos los estudiosos del Tercer Sector para quienes hay una clara correlación "entre una gestión profesionalizada de la comunicación y la existencia de un departamento de comunicación" (Balas, 2011:213).

La revisión bibliográfica confirma también que los Gabinetes de comunicación de las ONG están estructurados de manera similar a otras organizaciones: Comunicación interna y externa; Marketing y Publicidad; Relaciones con la sociedad/comunidad; y Comunicación de crisis (Ramírez, 1995; Almansa, 2011; Balas, 2011). A pesar de ello, los autores citados coinciden en destacar que cada ONG crea y organiza su Gabinete de comunicación y las funciones que quieren conseguir atendiendo a los objetivos generales propuestos por su organización, los diferentes públicos internos y externos y los recursos destinados a dicho departamento. Es relevante destacar en este punto que autores como Krohling inciden en sus investigaciones en la necesidad de que los departamentos de comunicación de las ONG nos sean réplicas de los empresariales puesto que su misión, visión y valores precisan un modo diferente de gestionar la relación con todos sus grupos de interés desde un enfoque más participativo (Krohling, 2013 a; Krohling 2013 b).

A pesar de que los recursos destinados a la comunicación aumentaron en las ONG hasta la crisis, algunos autores señalaban que el flujo comunicativo en muchas ONG todavía no circulaba suficientemente por "la falta de planificación en la comunicación interna, la escasa comunicación con las contrapartes y la casi inexistencia de relación con los movimientos sociales" (Erro \& Ventura, 2002: 107).

Otros estudios más recientes (Balas, 2011; Herranz de la Casa, 2009; González, 2006) apuntan, sin embargo, que los Gabinetes de comunicación entran a formar parte progresivamente de la estrategia de las ONG para mejorar su reputación y para el logro de sus fines a través de una comunicación bidireccional con sus diferentes públicos. En este sentido, para Balas (2011) la comunicación es una herramienta estratégica en la gestión de cualquier organización; pero cuando se trata de entidades en las que se conjuga la acción social y la eficiencia en procesos que tienen como fin último las personas, la trascendencia de una adecuada gestión de la comunicación adquiere una magnitud mayor.

La comunicación agrega valor al proyecto institucional. Por eso no es casualidad que la mayoría de las ONG con elevada visibilidad pública, estén dando cada vez más importancia a la comunicación, ya que ésta promueve el logro de su misión (Etkin, 2012:58). Además, en un entorno altamente competitivo (dado el número de organizaciones), por los mismos recursos (materiales y/o humanos; públicos y/o privados), la comunicación ayuda a crear una marca, favoreciendo la singularización y diferenciación entre las distintas entidades (Costa Sánchez, 2013). 


\section{La relación de las ONG con los medios de comunicación}

Uno de los vehículos utilizados por estas entidades para darse a conocer a nivel externo es la publicidad, pero otro no menos importante es la publicity, es decir, las relaciones con los medios de comunicación (Costa Sánchez, 2013).

Los medios de comunicación son un stackeholder más para las organizaciones por su capacidad de alcanzar a públicos amplios y diferenciados, segmentando los mensajes. Como señala Castillo (2011:5), "el poder de los medios consiste en preguntarse con qué efectividad pueden alcanzar objetivos sobre otras personas a voluntad de quienes los dirigen, poseen o controlan y quienes se valen de ellos para canalizar sus mensajes". Desde mediados del siglo XX existen diferentes investigaciones sobre los efectos sociales de los medios configurándose las principales teorías de los efectos cognitivos de los medios que inciden en su influencia en la conformación de actitudes. En este sentido, si atendemos a la Teoría de la Agenda setting, McCombs y Shaw (1972:176) apuntan que los medios "fuerzan la atención pública hacia ciertos temas, construyen las imágenes públicas de figuras políticas y presentan objetos sobre los que los individuos deben pensar, conocer o tener sentimientos" existiendo una relación entre el espacio concedido por los medios a los temas y la importancia que la gente cree que tienen los mismos. En esta línea, los medios de comunicación ejercen una influencia en la opinión pública como consecuencia del condicionamiento previo de lo que es o no ofrecido a su atención por los medios (Dader, 1990). Así la agenda mediática ejerce una influencia en las agendas pública e institucional (Dearing \& Rogers, 1996).

Otras teorías como la de la Tematización y Framing (Entman, 1993; Scheufele, 1999; Mc Combs \& Dixie, 1995) o la espiral del silencio de Noelle-Newman (2010), inciden también en los efectos de los medios. En este sentido, Luhmann (2000) remarcaba que los medios seleccionan los acontecimientos que se convertirán en noticias para, a través de ellas, construir los temas y mantenerlos mientras son de actualidad. Además, Goffman (1986) utilizaba la palabra frame para referirse a elementos con arreglo a los que se construyen las definiciones de una situación y McCombs alude a como los medios enmarcan sus contenidos y eso da lugar al proceso de framing que se refiere al origen de ciertos marcos y su difusión desde los medios masivos hacia el público (McCombs \& Dixie, 1995). Por otra parte, en cada paso del proceso de comunicación se toman decisiones donde se incluye o excluye información, se ignoran asuntos o se incluyen recursos para ilustrar los temas. Cada uno de estos pasos es, en esencia, la imposición de un encuadre. Según Entman (1993:52), "enmarcar es seleccionar algunos aspectos de una realidad percibida y hacerlos destacar en un texto comunicativo, de tal manera que se promueva la definición en particular de un problema".

Por ello, a pesar del gran desarrollo que han experimentado los denominados "medios sociales", actualmente las relaciones de las ONG con los medios tradicionales siguen siendo necesarias, ya que las apariciones mediáticas "facilitan la creación de una mayor sensibilización social hacia los problemas defendidos por estas organizaciones" (Castillo, 2007:196). Así, las ONG necesitan a los medios para llegar a la ciudadanía, sensibilizarla, educarla y concienciarla, pero además estas entidades necesitan la participación de los mass media para diferenciarse y ser competitivas en una sociedad cambiante. Según López Rey (2006: 153), "de cara a la sociedad en general es necesario controlar la información que se facilita y cuidar una imagen pública de la que depende en gran medida la captación de recursos, tanto económicos como humanos, y por tanto la legitimidad de la acción de la ONG". 
Según un estudio de la Fundación Hazlo Posible y la Comunidad de Madrid (2011) donde fueron consultadas 289 ONG españolas, uno de los grupos de interés prioritarios en los que se concentrarán los esfuerzos de trabajo de las ONG en los próximos años eran los medios de comunicación, apuntando como objetivos prioritarios: la transparencia, la captación de fondos e impulsar la participación social y movilización ciudadana.

Entre los antecedentes en el estudio de las relaciones con los medios de las ONG en España, González (2006:72-74) realiza un recorrido histórico desde la década de los 90, encontrando cinco fases: hasta 1992 la fase de inexistencia de conocimiento y relación donde las ONG casi nunca eran noticia siendo su labor desconocida para los periodistas para los que no eran fuente de referencia; de 1992 a 1993, la fase de autoridad como agentes humanitarios de desarrollo donde se inician los primeros contactos y se publican informaciones en los medios sobre cooperación y emergencia; desde 1994 hasta el 2000 la fase de climax de autoridad donde confluyen los intereses de ONG y medios que cuentan con secciones especializadas sobre solidaridad, aun cuando deben consolidarse como fuentes autorizadas de información; de 2000 a 2002 una fase de cuarentena de la autoridad donde aparecen movimientos críticos que cuestionan la fiabilidad de estas entidades por el afán de protagonismo o por polémicas por fraudes en la gestión de los recursos económicos, o polémicas sobre su neutralidad generando desconfianza hacia las ONG; y la última fase se denomina "de profesionalización comunicativa", donde las ONG apuestan por reforzar y profesionalizar sus departamentos de comunicación.

Otros autores (Ortega, 1994; Bel, 2004; Wilcox, 2012) han abordado los objetivos de la relación con los medios. En este sentido, Castillo (2007) apunta cómo las principales funciones de la gestión de las relaciones con los medios las siguientes: difusora de sus propuestas a los ciudadanos; movilizadora de adhesiones; facilitadora de cohesión entre sus miembros; de apoyo social, exhibiendo ante los poderes públicos a través de los medios el soporte social de las ONG; una función educativa; de sensibilización de la sociedad; de imagen, mediante apariciones positivas; monopolizadora, para mediante una especialización temática y legitimadora, conseguir -a través de la presencia en medios- legitimidad ante los poderes públicos, sus miembros, los ciudadanos y los propios soportes comunicativos.

Para potenciar la presencia en los medios de estas entidades, algunas organizaciones y autores proponían la necesidad de agruparse para dirigirse a los medios desde colectivos que proyecten mensajes unitarios. En esta línea, Jerez y Echart (2002:46) señalan que a veces "cometemos el error de dirigirnos a los medios uno a uno, con lo que provocamos una saturación". Además, apuntan que los medios en ocasiones no entienden por qué no hacemos un frente unitario para explicar cuestiones que son de interés general para todos los colectivos implicados, más allá de conseguir publicaciones pequeñitas (Jerez y Echart, 2002). Por otra parte, un estudio realizado por González (2005) centrado en el análisis de la gestión de la información difundida por las ONG y las noticias publicadas por los medios sobre ellas, ponía de manifiesto la necesidad de la profesionalización de los departamentos de comunicación de las ONG como una herramienta muy útil para contrarrestar imágenes sesgadas difundidas en los medios. Además, la misma autora se refería a las principales tácticas de relaciones públicas utilizadas por los gabinetes de comunicación de las ONG incluyendo, en los primeros lugares, varias orientadas a los medios: el comunicado de prensa como la herramienta más utilizada por estas organizaciones; las convocatorias y ruedas de prensa para informar sobre temas destacados, y el dossier de prensa para aportar una información más completa. 
Otros investigadores han abordado el papel de las ONG como fuentes informativas. En este sentido Herranz (2010) entiende que uno de los principales objetivos de los Gabinetes de comunicación de las ONG en sus relaciones con los medios es aparecer dentro de sus agendas de contenidos: hay que entrar en la agenda de los medios y superar las barreras del silencio para que el mundo de las ONG forme parte de las noticias sociales, económicas o internacionales. Para ello, el autor indica que las organizaciones tienen que ser más innovadoras y creativas al elaborar sus noticias debiendo potenciar herramientas más cercanas como entrevistas o conferencias de prensa frente al envío tradicional de notas de prensa (Herranz, 2010). Por su parte Lamy (2016) en su investigación, al analizar la presencia de las ONG como fuentes informativas, en su caso en medios informativos portugueses, confirma que existen diferencias en la cobertura que las agencias de noticias, los medios impresos, los radiofónicos y los audiovisuales les otorgan.

Es relevante en este apartado mencionar a investigadores como Sampario-Dias (2016), Murciano (2010) 0 Moeller (1999), que en sus estudios, además de analizar la presencia mediática de temáticas como la Cooperación, los Derechos Humanos, la Solidaridad, investigan también los hándicaps a los que se enfrentan los periodistas que trabajan en los medios de comunicación cuando quieren dar cobertura a estas temáticas. El conocimiento por parte de las ONG de estas dificultades a las que se enfrentan estos periodistas puede contribuir a mejorar la relación comunicativa que mantienen con ellos para aumentar su presencia.

Por otro lado, la revisión bibliográfica descubre un aumento en los últimos años de las investigaciones sobre la gestión de la comunicación en el ámbito on line en las ONG (Krohling, 2016; Krohling, 2013 c; González, 2014; Pinazo \& Nos, 2013; Marí, 2013; Arroyo \& Baños, 2013b; Ramil, 2012; Soria, 2011). En este sentido, algunos investigadores se refieren a las Salas on line como recurso para mejorar las relaciones entre los Gabinetes de las ONG y los medios. Para Soria (2011) las ONG son cada vez más conscientes de que el trabajo de los periodistas está marcado por la inmediatez, por lo que con esta herramienta tratan de facilitar su tarea proporcionándoles una información casi en tiempo real.

Algunos autores se refieren a la relación entre ONG y medios de comunicación aludiendo a que no ha sido fácil. En este sentido, Costa Sánchez (2013) alude a cómo las organizaciones ponen de manifiesto la falta de interés de aquéllos por la cooperación, la solidaridad o las noticias que ellas generan, acusando a los medios de no prestar atención ni dar cabida a las temáticas solidarias, así como de centrarse en el carácter espectacular o conflictivo del mensaje.

Desde la perspectiva de los medios, es reseñable el Informe de CONDGE (2007) sobre la percepción de los periodistas del trabajo de los gabinetes de las ONG. Dicho estudio destaca que el $90 \%$ de los periodistas las consideraba fuentes fiables de información, si bien el 63\% de ellos aseguraban que no reaccionaban igual cuando recibían información de una entidad grande frente a las pequeñas. El $73 \%$ incidió en el interés de los medios para difundir las informaciones referidas a las ONG, siendo la falta de espacio la causa de que no se conceda un tratamiento mayor. Por otro lado, aunque el $81 \%$ de los periodistas consideraba la relación con los gabinetes como buena, la mayoría incidía en que podían mejorar su trabajo con contenidos más originales y evitando la información publicitaria.

En general, los periodistas califican la relación con las ONG como escasa y puntual (Balas Lara, 2011, 261, 269). Ello es debido según los medios, a la falta de transparencia de las ONG y a un escaso conocimiento de las rutinas periodísticas y de profesionalización de los recursos dedicados a las relaciones con los medios (Costa Sánchez, 2013). 
A partir de la revisión de antecedentes en relación al objeto del estudio, podemos concluir la escasez de trabajos sobre su gestión con los medios tradicionales durante la crisis (González, 2013). El interés por analizar la relación con los medios en esta etapa radica en varios factores como la notoriedad aportada por los medios a las ONG, que ante las limitaciones económicas encuentran en la consecución de publicity un objetivo clave para evidenciar el incremento de necesidades cubiertas por las ONG y reivindicar la necesidad de recursos para frenar las desigualdades sociales. Además, en un momento de dificultad la credibilidad aportada por el tratamiento periodístico y su capacidad de influencia, puede ayudar a que el mensaje cale a la hora de sensibilizar y movilizar a la población en torno a las iniciativas desarrolladas por las ONG.

\section{Análisis y resultados}

Modelo de gestión de la comunicación de los Departamentos de comunicación de las ONG y consideración estratégica; influencia de la crisis

Cinco de los gabinetes son internos y uno externo (Casa Caridad), aunque Cruz Roja también contrata los servicios de Kantar Media (clipping regional). En todos los casos la evolución ha sido hacia un modelo de Gabinete de comunicación integral, aunque COCEMFECV y FEAPSCV comenzaron como Gabinete de prensa. Todos han señalado que la comunicación es considerada un factor estratégico (debido a una creciente concienciación por parte de sus Juntas de Gobierno) y la estrecha colaboración entre el Gabinete de comunicación y el resto de departamentos de la organización (en especial con Voluntariado y Captación de fondos).

\section{Plan de comunicación}

Cinco de las ONG elaboran sus primeros Planes de comunicación en la década del 2000, siendo pionero el de Cruz Roja (2001). Todas cuentan con un Plan de comunicación nacional y planes locales (salvo Casa Caridad que sólo trabaja en Valencia). En el 2008 todas disponen de dicho Plan menos COCEMFECV (en 2012), si bien sólo los de Cruz Roja y Cáritas cuentan con una estrategia a largo plazo (el resto anual).

Únicamente Cruz Roja y Cáritas realizan Planes con todas las fases desarrolladas (investigación, planificación, ejecución y evaluación) y el resto se centran principalmente en la fase de implementación (con calendario de ejecución). La evaluación es una asignatura pendiente para la mayoría pues no disponen de herramientas que midan con rigor los resultados de sus acciones comunicativas, destacando los casos de Cruz Roja y Casa Caridad (evaluación cuantitativa, económica y cualitativa parcial de las relaciones con los medios) y CEARPV, que evalúa sus acciones de comunicación en términos de movilización social y cambios producidos en las políticas públicas.

En relación a los cambios generados durante la crisis en la planificación de la comunicación (2008-2012), la investigación muestra lo siguiente:

- Cruz Roja ha reorientado su trabajo para atender a colectivos más vulnerables con campañas de llamamiento interno y externo ("Ahora + que nunca"). 
- Cáritas y Casa Caridad han tratado de aprovechar el incremento de requerimientos de los medios para visualizar más su trabajo y conseguir más recursos y voluntarios.

- CEARPV, COCEMFECV Y FEAPSCV han centrado sus acciones de comunicación para la denuncia de la disminución de ayudas económicas.

Área de relaciones con los medios

En la mayoría de las ONG la publicity es prioritaria frente a la publicidad, y los medios son considerados público clave. Casa Caridad es la que más dedicación presta a esta área (70\%), seguida de Cruz Roja (40\%), Cáritas (30\%) y FEAPSCV (25\%). CEARPV la considera prioritaria, pero su dedicación es discontinua y en los Gabinetes de COCEMFECV y FEAPSCV no es área prioritaria.

En cuanto a los recursos humanos, el responsable coincide en todas las ONG con el Dircom. Las entidades con más profesionales son Cruz Roja, Cáritas y Casa Caridad, que cuentan con personal contratado y voluntario, que han mantenido durante la crisis.

Las ONG que disponen de programa de campañas de prensa son Cruz Roja, Casa Caridad y Cáritas y el resto trabajan con objetivos pero sin dicha planificación. Casa Caridad es la que trabaja más proactivamente los temas con los medios y las demás son más reactivas, aprovechando las demandas de los periodistas. Los principales temas enviados a los medios se refieren a sus Programas de intervención social; les siguen las acciones de sensibilización y transmisión de valores, y a continuación proyectos de Cooperación internacional, Socorro, Emergencias y Empleo. Cruz Roja es la ONG que manifiesta una mayor diversificación de temas; Casa Caridad se ha posicionado como referente en datos de pobreza en Valencia; y CEARPV y Cáritas tratan de aprovechar oportunidades informativas.

Los principales encuadres de las informaciones enviadas son: "sensibilización social" y "divulgativo". Cáritas y CEARPV apuntan también el de "denuncia", y otros "captación de fondos" (CEARPV y COCEMFECV). Destaca el caso de CEARPV que con motivo de la crisis han cambiado su enfoque: de la "sensibilización" a la "denuncia y búsqueda de fondos".

5.2. Percepción de los periodistas: atención informativa a las ONG y temas priorizados

Un primer tema sobre el que hemos preguntado a los profesionales de los medios es si existe o no alguna política $\mathrm{u}$ orientación editorial en su periódico respecto a las informaciones relativas a ONGs y sus actividades. Cuatro de los periodistas entrevistados (El País, El Mundo, ABC y Las Provincias) señalan que no existe una orientación editorial en especial en sus periódicos, salvo el criterio de relevancia informativa y actualidad de los temas. No obstante, la mayoría reconocen haber incrementado su interés hacia las ONG y los movimientos sociales en 2012, debido a la repercusión de la crisis y al aumento de notas de prensa recibidas en torno al tema.

Por otro lado, algunos periodistas consideran que no siempre prestan la suficiente atención a las entidades del tercer sector debido principalmente a la falta de espacio en el medio o la actualización de contenidos ya señalada. En el caso de El Mundo, su periodista explica otro motivo distinto por el cual son más bien reactivos a este tipo de informaciones: consideran que la gente muestra menos interés hacia esta cuestión, al ser la crisis un tema tan recurrente en los medios. En $A B C$ reconocen que tampoco son proactivos con estas informaciones, sobre todo por falta de personal disponible en la redacción para cubrirlas. 
Levante es el único medio que siempre ha apostado de manera positiva por este tipo de contenidos, dedicando secciones especializadas desde hace años: los semanales "Solidarios" e "Integrados"; el suplemento "Juntos"; diversos espacios en la edición del fin de semana. Las Provincias apunta que en ocasiones también deciden publicar algunas informaciones relativas a las ONGs en la edición de fin de semana, que admite más espacio. Y en todo caso señalan que a veces intentan "valencianizar la información"; es decir, trasladarla y adaptarla a la situación del entorno local.

\section{Las ONG como fuentes}

Al preguntar a los periodistas si consideran a las ONG como fuentes fiables y relevantes, casi todos manifiestan mantener trato frecuente con los Gabinetes de estas entidades, y los consideran fuentes habituales para contrastar sus informaciones. No obstante, destacan que confían más en las ONG de mayor tamaño, trayectoria, así como base social consolidada por su experiencia (aportan mayor conocimiento de los temas), elevada cantidad de personas atendidas y representatividad de su trabajo con determinados colectivos.

En el caso de El País valora positivamente la existencia de relaciones fluidas y mantenidas en el tiempo con los mismos profesionales de la comunicación de estos Gabinetes. Y $A B C$ señala que no existe trato personal con la mayoría de ONG por lo señalado sobre su escasez de personal.

En cuanto a la calidad de la respuesta por parte de las ONG a los requerimientos de los medios, los profesionales de El País, Levante y Las Provincias señalan que normalmente es positiva. El diario El Mundo apunta sin embargo que a veces desde los Gabinetes podrían ajustar mejor los tiempos; es decir, responder con más agilidad a requerimientos que se hacen desde la redacción, como fotografías o imágenes, ampliación de la información, etc. El profesional de $A B C$ señala que estas entidades convocan actualmente menos ruedas de prensa, pensando que no tienen interés para los medios, o que no acudirán por falta de tiempo y recursos. En su opinión, podrían aumentar la atención e interés de los medios hacia ellas si trabajaran más algunos temas u ofreciendo entrevistas más allá de las notas de prensa o proporcionando más datos.

Las ONG van preparando también a distintos miembros de su organización (directivos, coordinadores de proyectos, voluntarios, etc.) para intervenir como portavoces que puedan ser entrevistados por los medios, pues éstos señalan que les resulta imprescindible contactar con personas que tengan un conocimiento profundo de los temas tratados para poder comentarlos analizando causas, aportando más datos, etc. El testimonio además cobra especial importancia si se tiene en cuenta el tipo de acciones desarrolladas por estas entidades.

\section{Temas solicitados a las ONG y enfoques predominantes}

Es unánime la respuesta de los encuestados respecto a los temas que se reciben con más frecuencia en las redacciones, enviados por los Gabinetes; destacan especialmente: los que evidencian desigualdades sociales relacionadas con pobreza y exclusión, inmigración, discapacidad, y especialmente aquellos en que denuncian situaciones injustas. En cambio, todos los periodistas -salvo el de Levante- señalan que los contenidos y temas recibidos de las ONG no se refieren mayoritariamente a dichos asuntos de interés, sino a los fines y 
actividades de estas entidades; en segundo lugar, a la solicitud de colaboración (económica y/o voluntaria); y sólo en tercer lugar, a la denuncia. Para el profesional de El Mundo, mucha información recibida de las ONG va "directamente a la papelera por falta de interés informativo", y aclara que realmente los temas no es que carezcan de interés en sí, sino que a veces no tienen el enfoque de actualidad que consideran necesario para publicarlos.

Los periodistas de El Mundo y Las Provincias añaden que les interesan en especial testimonios de usuarios o voluntarios, y Levante se inclina por proyectos de cooperación internacional, además de que muchas de las informaciones sobre el sector tienen cabida -como hemos señalado anteriormente- en secciones especiales.

En cuanto al encuadre de las informaciones, la mayoría de los medios entrevistados intentan enfocarlas en clave divulgativa, de denuncia (sobre todo El País y El Mundo) y sensibilización (especialmente Levante). En general los profesionales de los medios recomiendan a las ONG ser más incisivos y denunciar situaciones, pues "a veces se les pasan cosas que serían noticia y gozan de credibilidad" (El País). También proponen, ante la escasez de espacio físico de los medios off line, aprovechar internet para publicar muchas de sus informaciones.

\section{Tratamiento informativo obtenido por las ONG}

\section{Cobertura en prensa}

La cobertura obtenida por las seis ONG en los medios y periodo analizados ha sido de 360 textos (Gráfico 1), siendo superior en 2012. Cáritas, Cruz Roja, Casa Caridad y FEAPSCV han experimentado un incremento de su cobertura en 2012, disminuyendo solo en los casos de CEARPV y COCEMFECV.

Gráfico 1: Cobertura obtenida por las ONG

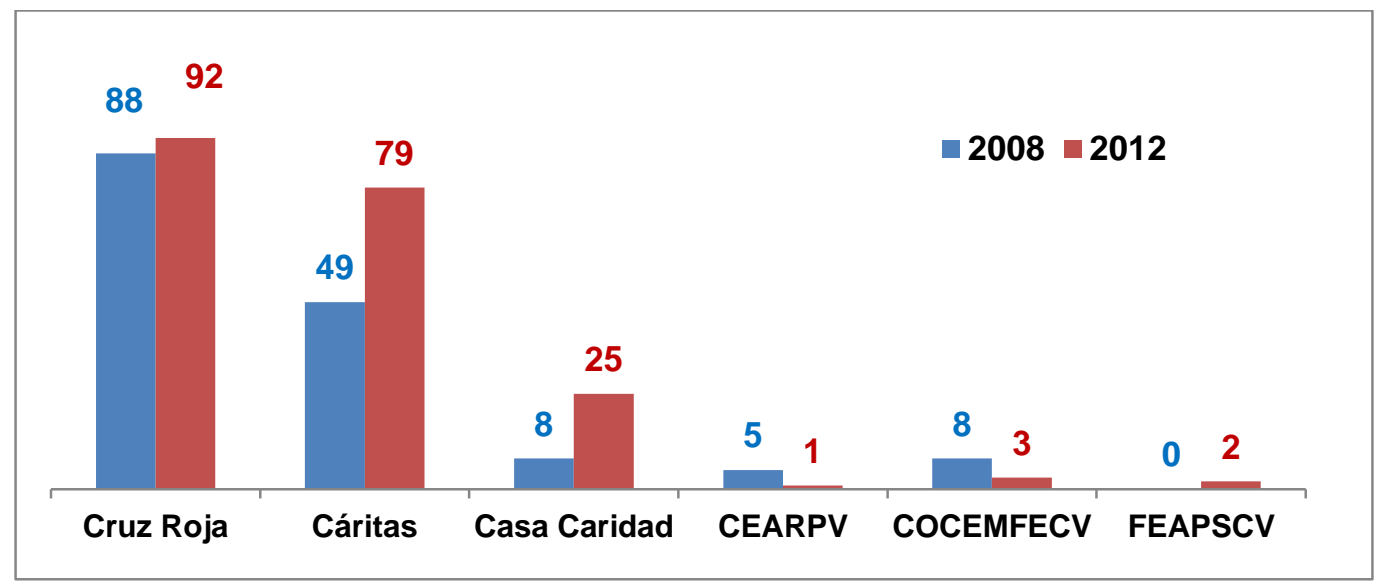

Fuentes, temas, personajes, mención de la crisis y encuadres predominantes

En el 2012 el mayor porcentaje corresponde a informaciones donde las ONG no aparecen citadas como fuentes, a pesar de que la cobertura informativa alcanzada por las ONG este año es mayor. Las ONG más citadas como fuente principal (Tabla 1) han sido Cáritas y Cruz Roja (en 2008) y Casa Caridad (2012). 
Tabla 1: Fuente principal citada

\begin{tabular}{|l|r|r|}
\hline \multicolumn{1}{|c|}{ Fuente principal citada } & \multicolumn{1}{c|}{2008} & \multicolumn{1}{c|}{2012} \\
\hline $\begin{array}{l}\text { No hay fuentes principales } \\
\text { mencionadas }\end{array}$ & $19,6 \%$ & $30,2 \%$ \\
\hline Fuentes oficiales (instituciones) & $20,9 \%$ & $19,3 \%$ \\
\hline Cruz Roja & $12 \%$ & $7,4 \%$ \\
\hline Cáritas & $12 \%$ & $9,9 \%$ \\
\hline Casa Caridad & $3,2 \%$ & $4,5 \%$ \\
\hline CEARPV & $1,9 \%$ & $0 \%$ \\
\hline COCEMFECV & $3,8 \%$ & $1,7 \%$ \\
\hline FEAPSCV & $6,3 \%$ & $0,4 \%$ \\
\hline Otras ONGS & $1,9 \%$ & $4,0 \%$ \\
\hline Periodista/medio de comunicación & $5,7 \%$ & $4,5 \%$ \\
\hline Fuentes oficiales (políticos) & $7,6 \%$ & $9,4 \%$ \\
\hline Ciudadanos & $1,3 \%$ & $5,4 \%$ \\
\hline Beneficiarios ONGs & $0,4 \%$ & $1,2 \%$ \\
\hline Personajes cierta relevancia públicas & $3,2 \%$ & $2 \%$ \\
\hline Otros & & $1,7 \%$ \\
\hline
\end{tabular}

La temática principal de los textos (Tabla 2) ha sido el trabajo de las ONG, destacando "labor asistencial de necesidades básicas", "rescate y emergencias" y "captación de fondos/ayudas" (incremento en 2012).

Tabla 2: Temática principal

\begin{tabular}{|l|r|r|}
\hline \multicolumn{1}{|c|}{ Temáticas principales } & \multicolumn{1}{c|}{2008} & \multicolumn{1}{c|}{2012} \\
\hline Labor asistencial necesidades básicas & $15,2 \%$ & $15,8 \%$ \\
\hline Rescate y emergencias & $19,6 \%$ & $12,9 \%$ \\
\hline Captación fondos/concesión ayudas & $10,1 \%$ & $13,4 \%$ \\
\hline Formación miembros de la entidad & 0 & $2 \%$ \\
\hline Formación colectivos de riesgo & $4,4 \%$ & $1,5 \%$ \\
\hline Mediación institucional & $3,2 \%$ & $1 \%$ \\
\hline Formación institucional ONG & $1,3 \%$ & $2 \%$ \\
\hline Otros temas relativos ONG & $12 \%$ & $13,4 \%$ \\
\hline Otros temas no relacionados directamente & $34,2 \%$ & $38,1 \%$ \\
\hline con ONG & & \\
\hline
\end{tabular}

Los principales personajes sujetos de las informaciones sobre las ONG son los "inmigrantes y/o minorías raciales", aunque en 2012 disminuyen incrementándose la presencia de "ciudadanos", "familias" y "desempleados" (relacionado con la crisis), seguidos de "políticos o responsables de instituciones públicas" 
(que se han pronunciado públicamente o intervenido como consecuencia de la crisis). Cáritas y Casa Caridad incrementan su presencia como sujetos informativos en el 2012 (posiblemente relacionado con el incremento su trabajo durante la crisis).

Se ha analizado también la mención expresa de la crisis en los textos, constatando que aunque ambos años es mayor el número de textos que no la mencionan, en 2012 se ha producido un incremento del 20,3 \% de los artículos que la nombran.

En cuanto a los enfoques otorgados por los diarios a las informaciones (Gráfico 2), en 2012 se detecta una reducción del frame "divulgativo" (mayoritario) en favor de "denuncia social", "político" y "económico", predominando éstos en casi un $60 \%$ de las informaciones.

Gráfico 2: Frames priorizados

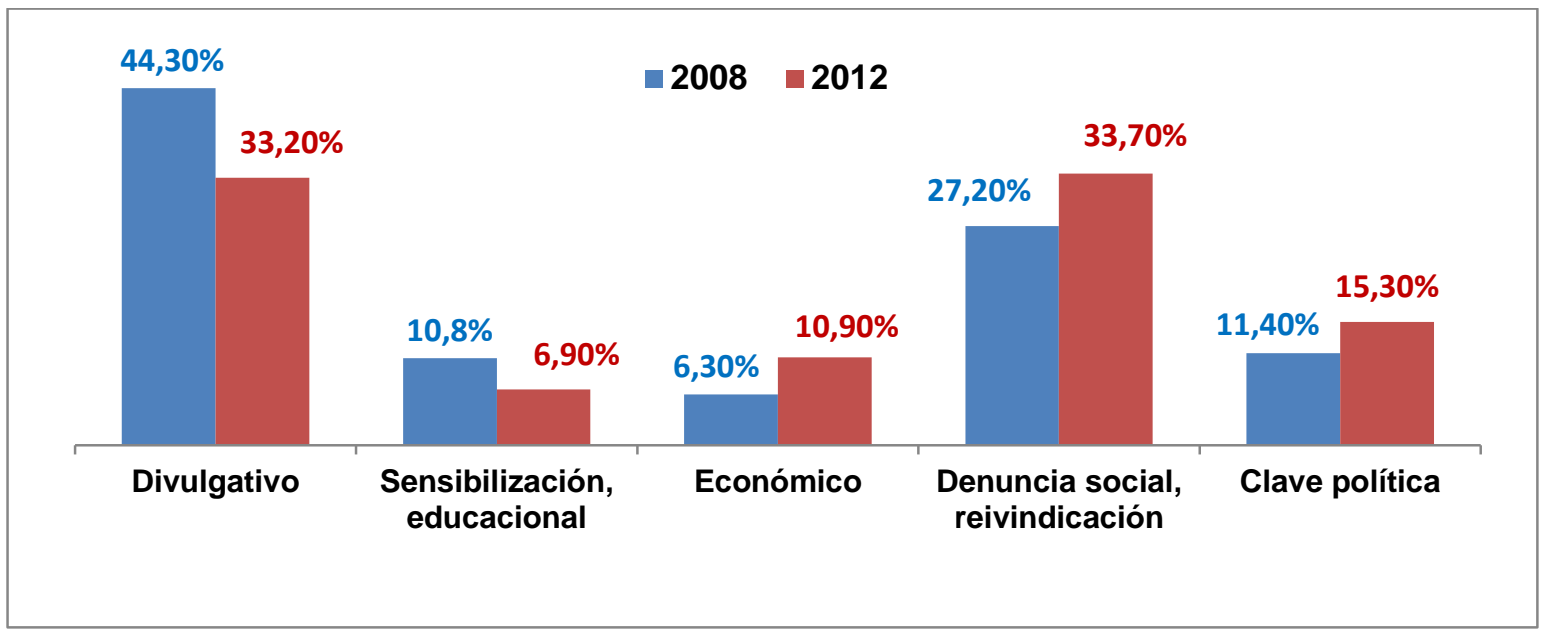

\section{Conclusiones}

La principal conclusión obtenida en esta investigación es que la crisis ha influido tanto en la gestión de la comunicación de las ONG, como en la percepción y tratamiento periodístico de los temas sociales en los medios, más receptivos hacia la labor de las entidades del Tercer Sector, generándose oportunidades informativas que las ONG no han rentabilizado totalmente.

Por un lado, Cruz Roja, Cáritas y Casa Caridad han sido las ONG que han sabido aprovechar la crisis para incrementar su cobertura mediática, reorientando los objetivos y temáticas de sus Planes de comunicación y beneficiándose del aumento de requerimientos por parte de los medios. No obstante, si atendemos a las temáticas y encuadres, descubrimos en los medios una mayor atención hacia los temas y frames relativos a la denuncia de las desigualdades, que las ONG no han aprovechado, priorizando la mayoría la divulgación de su labor frente a la denuncia de injusticias.

Esta conclusión se fundamenta en las siguientes inferencias obtenidas en relación a los objetivos de la investigación:

1. En relación al objetivo 1 (conocer el modelo de gestión de la comunicación de las ONG y el peso de sus relaciones con los medios) se concluye que las seis ONG gestionan su comunicación de forma 
profesional, predominando un modelo de Gabinete interno de comunicación integral con recursos limitados que se mantienen durante la crisis. Sin embargo, encontramos diferencias significativas en la gestión de la comunicación durante la crisis, destacando buenas prácticas comunicativas en Cruz Roja, Cáritas y Casa Caridad:

- Aunque todos los Gabinetes han mantenido durante la crisis una planificación y orientación estratégica de su comunicación, sólo encontramos Planes a largo plazo en Cruz Roja y Cáritas.

- Todas las ONG consideran a los medios un público clave con motivo de la crisis, aunque no todas los priorizan, y son Cruz Roja, Cáritas y Casa Caridad las que destinan más recursos a la gestión de las relaciones con los medios.

- Estas mismas entidades son las que han adaptado mejor los objetivos de sus Planes de comunicación, siendo las que mejores resultados han obtenido en cobertura y las más citadas durante la crisis.

- Los encuadres con los que la mayoría de las ONG han enfocado sus temas han sido la sensibilización de la ciudadanía y la divulgación de sus proyectos, siendo menos las informaciones de denuncia sobre las desigualdades provocadas por la crisis. Así, aunque Cruz Roja, Cáritas y Casa Caridad, han reorientado los temas durante la crisis, no han dado prioridad al frame de denuncia, a pesar de que cómo demuestra esta investigación era una de los enfoques que los periodistas hubieran tenido mayor predisposición a publicar.

- Por otra parte, a pesar de que todas las ONG se han visto sometidas a una reducción de ingresos para atender a sus crecientes necesidades, la denuncia de este hecho no ha sido destacada por las ONG con mayor cobertura (Cruz Roja, Cáritas y Casa Caridad). CEARPV, COCEMFECV y FEAPSCV, si han priorizado la denuncia de la disminución de ayudas pero su presencia ha sido menor.

2. En cuanto al segundo y tercer objetivo del trabajo (conocer la relación de los periodistas con las organizaciones del Tercer Sector y los cambios en el tratamiento informativo de las ONG en la prensa (en 2008 y 2012), las principales conclusiones son:

- La crisis ha marcado el tratamiento periodístico de las ONG; por una parte, los medios han estado más receptivos hacia los temas sociales y hacia el trabajo de las entidades del Tercer Sector -lo que podemos considerar una buena práctica informativa- y por otra, hemos constatado que han sido principalmente Cruz Roja, Cáritas y Casa Caridad las ONGs que más han incrementado su presencia durante la crisis.

- No obstante, aunque casi todos los periodistas confirman su interés por acudir a las ONG durante la crisis -coincidiendo con un incremento de las notas de prensa recibidas de éstas- el análisis de los textos muestra que los medios les han otorgado un menor protagonismo como fuentes, en favor de una mayor presencia de los beneficiarios de estas entidades.

- En general los periodistas achacan la escasa presencia que estas entidades tienen normalmente en los medios a la falta de espacio o de actualidad de los temas, animando a los Gabinetes a aprovechar las ediciones on line, actualizar su información y ser más incisivos. También destacan 
que confían más en las ONG de mayor tamaño, trayectoria y base social, así como por la representatividad de su trabajo con determinados colectivos.

- $\quad$ No obstante, todos los periodistas (menos el de Levante) señalan que las ONG podrían ser más incisivas en sus temas a la hora de denunciar situaciones injustas, ya que los contenidos recibidos mayoritariamente de las ONG suelen hacer referencia principalmente a sus fines y actividades, estando los medios más interesados en las desigualdades sociales relacionadas con la pobreza, la exclusión o inmigración.

- En cuanto a las temáticas y personajes aparecidos en los medios, se descubre una mayor presencia de sujetos vulnerables ante la crisis en 2012 (ciudadanos, familias, desempleados, personas con discapacidad), lo que parece apuntar a la visualización de los "nuevos pobres" señalados en la Introducción. También en el 2012 la crisis ha sido mencionada explícitamente en un 20\% más de informaciones, lo que manifiesta que ha marcado la agenda mediática de las entidades del Tercer Sector.

- Cruz Roja, Cáritas y Casa Caridad han sido las ONG capaces de visibilizar el incremento de necesidades cubiertas y de beneficiarios atendidos como consecuencia de la crisis (mediante un mayor número de notas de prensa enviadas a los medios y una mayor presencia mediática).

- En línea con lo anterior, las ONG han conseguido que se destaque el enfoque divulgativo de su labor, pero hay un cambio de tendencia en 2012 aumentando las informaciones con frame de "denuncia social", a través del que los medios han tratado de visibilizar las injusticias incrementadas por la crisis, lo que manifiesta una buena práctica por parte de los medios y una oportunidad que podría haber sido mejor aprovechada por todas las ONG.

A partir de las conclusiones apuntadas, queda validada la primera hipótesis del trabajo al confirmar que la crisis económica, si bien no ha producido cambios en el perfil y RRHH de los Gabinetes de comunicación de las ONG analizadas, sí ha influido en la reorientación y planificación de los objetivos de comunicación, priorizando la mayoría a los medios como un grupo clave.

También quedaría validada la segunda hipótesis -dada la influencia de la crisis en las ONG- provocando cambios en el modelo informativo y tratamiento periodístico hacia estas entidades, con una mayor atención mediática hacia los temas sociales y las entidades del Tercer Sector, mostrándose los medios más receptivos para dar a conocer la labor que desarrollan con colectivos en riesgo de exclusión, así como comprometiéndose más con la denuncia de las desigualdades. Esto ha supuesto la generación de nuevas oportunidades para las ONG -aunque su presencia como fuentes no haya sido elevada-, confirmándose en la mayoría un incremento de su visibilidad en los medios durante la crisis.

No obstante, los resultados obtenidos en esta investigación no permiten confirmar si, en el aumento de cobertura de la crisis donde las ONG son noticia, tuvo más peso la mayor proactividad de los periodistas por informar sobre este ámbito noticioso, o una mayor iniciativa difusora de contenidos al respecto por parte de estas entidades. Los datos obtenidos apuntan más bien a que fue la combinación de ambos factores lo que conllevó un aumento de la presencia de estas temáticas durante la crisis en los medios analizados.

La investigación también confirma que compartir el conocimiento de las necesidades informativas entre los periodistas y los profesionales que trabajan en los departamentos de comunicación, hubiera facilitado la identificación de temáticas y enfoques relevantes para ambos actores, mejorando cuantitativa y 
cualitativamente la cobertura mediática. Sirvan de ejemplo las noticias con enfoque de "denuncia social" que ambos estaban interesados en difundir y que no obtuvieron una mayor presencia en la prensa en el caso de algunas entidades analizadas. Este hecho, en parte, pudo ser debido a que aunque había un interés manifiesto por parte de los periodistas sobre esa temática, varias de las ONG analizadas no supieron adaptar los contenidos difundidos hacia ese enfoque, obteniendo una mayor cobertura mediática las entidades que supieron identificar esa oportunidad informativa adaptándose a las necesidades de los medios.

\section{Bibliografía}

Almansa, A. (2004). Historia de los Gabinetes de comunicación en España. Historia de la Comunicación Social, 9, 5-21.

Almansa, A. (2011). Del Gabinete de prensa al Gabinete de comunicación: la dirección de la comunicación en la actualidad. Zamora: Comunicación Social.

Arroyo, I. \& Baños, M. (2013a). La eficacia de la comunicación de las organizaciones del Tercer Sector en los vídeos emitidos a través de Youtube. Historia y Comunicación Social. Vol. 18 No Especial Octubre, 615-626.

Arroyo, I. \& Baños, M. (2013b). Tendencias de la comunicación del Tercer Sector en la web 2.0. Análisis retórico de los tropos. Icono 14, volumen 11 (2), 331-356

Arroyo, I.; Baños, M. \& Van-Wyck C. (2013c). Análisis de los mensajes audiovisuales del Tercer Sector en YouTube, Revista Latina de $\quad$ Comunicación Social, no 68, 328-345.

Balas, M. (2011). La gestión de la comunicación en el Tercer Sector. Cómo mejorar la imagen de las ONG. Madrid: ESIC

Baraybar, A. (2009). Conectando valores. Las nuevas estrategias de la comunicación online en el Tercer Sector, Icono 14-13, 67-55

Bardín, L. (1986). Análisis de contenido. Madrid: Akal Universitaria.

Bel, J.I (2004). Comunicar para crear valor. Pamplona: EUNSA.

Belzunegui, A. (coord), (2012). La socialización de la pobreza en España: género, edad y trabajo en los riesgos frente a la pobreza, Icaria Antrazyt.

Berganza, M. R. y Ruiz, J. A. (2005). Investigar en comunicación. Madrid: McGraw-Hill.

Cantó, O. (2011). Menores en riesgo de pobreza en España, Crítica, no. 976, 2011, 14-20.

Caritas, (2011). Cáritas ante la crisis. VI Informe sobre las demandas atendidas a través de la red confederal de Acogida y Atención primaria. Observatorio de la Realidad.

Castillo, A. (2007). Relaciones públicas en organizaciones no gubernamentales, Sphera pública: Revista de Ciencias Sociales y Comunicación, 7, 193-210.

Castillo, A. (2011). Los medios de comunicación como actores sociales y políticos. Razón y Palabra, № 75, 1-21.

CONGDE (2007). Informe sobre los medios de comunicación y las ONGD. Situación actual y retos. (http//www.congde.org/contenidos/descargar/attachedfiles/962/original?1363750391)

CONGDE, (2014). Informe encuesta del estado del sector 2014. (http://www.congde.org/contenidos/datossituacion-de-las-ong-de- desarrollo-miembros-de-la-coordinadora) 
Costa Sánchez, C. (2013). ONGDs y la prensa: Estrategias de territorialización en Galicia. Sphera Publica, 13, vol. I, 136-165.

Dader, J. L.(1990). Las Teorías contemporáneas; la canalización o fijación de la agenda por los medios; Teorías de la transición. En Muñoz, A.; Monzón, C.; Rospir, J. I. y Dader, J. L. (Eds) (1990). Opinión pública y comunicación política. Madrid: Eudema. 303-304

Dearing, J. \& Rogers, E. (1996). Agenda-Setting. California: SAGE Publications.

Duran, P. \& Fernández, M. B. (2010). "La comunicación en las organizaciones del tercer sector. Revista Latina de Comunicación Social, no 65, 595-603.

Etkin, E. (2012). Comunicación para organizaciones sociales: de la planificación a la Acción. Buenos Aires: La Crujía.

Erro, J. \& Ventura, J. (2002). El Trabajo de comunicación de las ONGD en el País Vasco. Bilbao: Hegoa.

ESADE, PWC \& Fundación La Caixa (2013) Estudio sobre el presente y futuro del Tercer Sector social en un entorno de crisis, Barcelona.

Entman, R. (1993). Framing: toward clarification of a fractured paradigm. Journal of Comunication, 43(4), 51-58.

Fundación Hazloposible y Comunidad de Madrid (2011). Informe sobre las necesidades comunicativas de las ONG. Valencia. Consultora Bidea.

Fundación Luis Vives (2013). Informe sobre el impacto de la depresión económica en el Tercer Sector de Acción social. Revista española del Tercer Sector de la Fundación Luis Vives, 23 (11-250).

FUNDAR, (2011). Informe-Evaluación sobre la capacidad comunicativa del sector asociativo en la Comunidad Valenciana, (http://www.fundar.es/descargas/varios/estudio_comunicacion_definitivo.pdf)

García, B. (2006). Aproximación teórica a la comunicación en el tercer sector. La necesaria reclasificación de la comunicación organizacional, TELOS, Cuaderno de Comunicación e Innovación, nº 69, 51-59.

Goffman, E. (1986). Frame analysis: an essay on the organization of experience. Boston: Northeastern University Press.

Gómez, C, (2013). ONG's en crisis y crisis en las ONG's un fin de ciclo en el "oenegeísmo" en España, Temas para el debate, №. 221, 23- 25.

González, H. (2005). Estrategias de comunicación e impacto mediático de las ONGS. Tesis Doctoral. Pamplona: Universidad de Navarra.

González, H. (2006). Estrategias de comunicación en las ONG de desarrollo: Departamentos, funciones e impacto en los medios. Madrid: CIDEAL.

González, H. (2013). Radiografía de la presencia de la solidaridad, la cooperación, el desarrollo, y las ONGD en la prensa regional navarra, Estudios sobre el Mensaje Periodístico, Vol. 19, Núm. 1, 147-164.

González, H. (2014). Oportunidades e implantación de las herramientas de la web social en la gestión comunicativa de las ONGD, Observatorio (OBS*) Journal, vol.8 - n4, 17-38.

González, M. I. (2010). Comunicación para la Solidaridad: las ONG y el papel de la comunicación social y periodística en la sensibilización de la opinión pública y el desarrollo. (Tesis de doctorado, Universidad Complutense de Madrid). (http://eprints.ucm.es/11633/2

Gutiérrez-Domènech, M. (2013). Radiografía poscrisis de la pobreza en España, Informe Mensual- La Caixa, No. 
(http://www.lacaixaresearch.com/documents/10180/55077/IM+366+Marzo+2013+CAST.pd f/25d63542-877f-4533-aa8d- f044defdd6ca)

Herranz de la Casa, J. M (2009). Comunicación y transparencia en las organizaciones sociales. ICONO, Revista de comunicación y Nuevas tecnologías, 13, 172-194.

Herranz de la Casa, J. M. (2010). La comunicación y la transparencia en las organizaciones no lucrativas. (Tesis de doctorado, Universidad Complutense de Madrid). (http://eprints.ucm.es/11539/)

INE (2012). Encuesta de Condiciones de Vida (Datos provisionales 2012). (http://www.ine.es/)

INE, (2014). España en cifras 2013. (http://www.ine.es/prodyser/espa cifras/2013/\#1)

Jerez, A. y Echart, E. (2002). ONGs y comunicación: entrevista a Teresa Burgui, Chusa Lamarca y Carlos Ballesteros. El viejo topo, 46-53.

Kayser, J. (1982). El diario francés. Barcelona: A.T.E.

Krohling Peruzzo, C. M. (2013, a): Fundamentos teoricos das relacoes públicas e da comunicacao organizacional no terceiro setor: perspectiva alternativa. Revista Famecos - Midia, Cultura E Tecnologia, (1), 89.

Krohling Peruzzo, C. M. (2013, b): Movimentos sociais, redes virtuais e mídia alternativa no junho em que "o gigante acordou"(?). Matrizes, 7(2), 73-93.

Krohling Peruzzo, C. M. (2013 c). Movimentos sociais, redes virtuais e mídia alternativa no junho em que "o gigante acordou"(?). Matrizes, 7(2), 73-93.

Krohling Peruzzo, C. M. (2016). La comunicación en los movimientos sociales y el Derecho a la Comunicación: Señales de un derecho de ciudadanía de quinta generación. Commons: Revista De Comunicación Y Ciudadanía Digital, 5(2), 10-36.

Lamy, S. (2016). As ONG como fontes de informação nos media - um olhar sobre o discurso jornalístico não governamental na RTP, TSF, Público e agência Lusa. Observatorio (OBS*), (4), 116.

López, J.A (2001), Solidaridad y mercado, A Coruña: Netbiblo.

López Rey, José Antonio (2006): El tercer sector y el mercado: conflictos institucionales en España. Madrid: Centro de Investigaciones Sociológicas (CIS).

Luhmann, N. (2000). La realidad de los medios de masas. Barcelona: Anthropos Editorial.

Marfil, R. (2013). Interactividad digital y estrategias narrativas en la publicidad audiovisual de Manos Unidas y Unicef. Historia y Comunicación Social. Vol. 18 No Especial Octubre, 169-181

Marí, V. (2013). Comunicación, desarrollo y cambio social en España: entre la institucionalización y la implosión del campo, COMMONS - Revista de Comunicación y Ciudadanía Digital, Vol. 2, 3, 40-64.

Martínez, I. (2013). Las ONG's españolas ante la crisis: ¿continuidad o transformación? Temas para el debate, 221, 37-39.

McCombs, M y Dixie, E. (1995). Los temas y los aspectos. Explorando una nueva dimensión de la agenda setting. Comunicación y Sociedad, Vol. VIII(1) 7-32.

McCombs, M. \& Shaw, D. (1972). The Agenda-Setting Function of the Mass media, Public Opinion Quarterly, $36,176-187$.

Moeller, S. D.( 1999) Compassion Fatigue. London: Routledge.

Murciano, M. (dir), La prensa y la cooperación internacional, Comunicación social, Zamora, 2010.

Noelle-Neumann, E. (2010). La espiral del silencio. Opinión pública: nuestra piel social. Barcelona: Paidós. 
Nos E; Iranzo A \& Farné A. (2012). La eficacia cultural de la comunicación de las ONGD: los discursos de los movimientos sociales actuales como revisión, Cuadernos de Información y Comunicación, 17, 209-237.

Ortega, M. L. (1994). Las ONGD y la CRISIS del Desarrollo. Madrid: IEPALA.

Ortega, E. y Peñalosa, J. (2012). Claves de la crisis económica española y retos para crecer en la UEM. Serie Documentos Ocasionales, № 1201. Madrid: Banco de España.

OTS, (2009) La crisis y el tercer sector: una oportunidad para la transformación social. Una visión a partir del consejo asesor de investigación del OTS, (http://www.observatoritercersector.org/pdf/publicacions/2009-03 crisis cast.pdf)

Paricio Esteban, Pilar; Rodríguez-Luque, Cristina y Rabadán, Ma Jose (2012). Tratamiento del consumo de alcohol y su prevención en la prensa española desde la perspectiva del framing: El País, El Mundo, ABC y la Razón. Revista Latina de Comunicación, 67. 3a Época. Págs. 322-346.

Pinazo, D. \& Nos, E. (2013). Developing Moral Sensitivity Through Protest Scenarios in International NGDOs Communication. CommunicationResearch, 1-24.

Piñuel, J. L. y Gaitán, J. A. (1998). Metodología general. Conocimiento científico e investigación en la comunicación social. Madrid: Síntesis.

Plataforma de ONG (2015). El tercer sector de acción social en 2015: Impacto de la crisis. Noviembre de 2015.

Público (2013). 2012, el año en que la crisis empujó a los ciudadanos a la calle. Público, 12 de enero de 2013. http://www.publico.es/espana/2012-ano-crisis-empujo-ciudadanos.html. Consultado el 17 de mayo de 2018.

Ramil, X. (Coord) (2012). ParadigmaTICås comunicación y cultura digital en las ONGD de desarrollo, Madrid, CONGDE.

Ramírez, T. (1995). Gabinetes de comunicación. Barcelona. Bosch.

Regadera, E. (2014). Gestión de la Comunicación de las ONG en Valencia en tiempos de crisis. Análisis de las relaciones con los medios y el tratamiento en prensa de Cruz Roja, Cáritas, Casa Caridad, CEARPV, COCEMFE, FEAPSCV (2008 y 2012). Tesis doctoral.

Rocha, F. y Aragón, J. (2012). La crisis económica y sus efectos sobre el empleo en España. Gaceta Sindical, 2012. http://docpublicos.ccoo.es/cendoc/035344CrisisEconomicaEfectos.pdf. Consultado el 17 de mayo de 2018.

Rojas, O. (2005). Relaciones públicas: La eficacia de la influencia. Madrid. ESIC.

Ruiz-Giménez, M. (2013). El futuro de las ONG's, un reto en definición. Temas para el debate, 221, $19-22$.

Scheufele, D. (1999). Framing as a theory of media effects. Journal of Communication, 49, 101-120.

Sampario Dias, S. (2016). Reporting human rights. New York : Peter Lang, 2016.

Soria, M.M (2011). La interacción de los públicos en las ONG 2.0: El estado actual de la comunicación social, Revista internacional de relaciones públicas, 2, 175-195.

Stempel, G. H. \& Westley, B. H. (1981). Research Methods in Mass Communication. Englewood Cliffs: Prentice Hall.

Vallés, M. (2002). Entrevistas cualitativas. Madrid: Centro de Investigaciones Sociológicas.

Wilcox, D.; Cameron, G; Xifra, J (2012). Relaciones públicas. Estrategias y Tácticas. Madrid. Pearson Addison Wesley. 